\title{
Mental Health Act 1983: use of urgent treatment in clinical practice
}

\author{
Rashmi Yadav, Anthony S. Zigmond ${ }^{1,2}$
}

The Psychiatrist (2013), 37, 156-159, doi: 10.1192/pb.bp.112.038414

${ }^{1}$ Leeds Partnerships NHS Foundation Trust; ${ }^{2}$ University of Leeds

Correspondence to Rashmi Yadav (rashmi.yadav@nhs.net)

First received 2 Jan 2012, final revision 25 Sep 2012, accepted 10 Oct 2012
Aims and method To understand circumstances in which urgent treatment provisions are used in clinical practice, by means of a retrospective study. A list of patients to whom Sections 62,64B and 64G of the Mental Health Act 1983 were applied during the 1-year study period was obtained from the information technology department. Case notes were traced for detailed information on the circumstances of use of these provisions.

Results The most common reason for urgent treatment was to continue the established treatment plan rather than to start a new treatment (45\% Section 62, $84 \%$ Section 64 ). The urgent treatment provisions were most commonly used because of a delay in examination by a second opinion appointed doctor in $45 \%$ cases for Section 62 and 84\% cases for Sections 64B and 64G.

Clinical implications This is probably unlawful use of the urgent treatment provisions.

Declaration of interest None.
The Mental Health Act 1983 for England and Wales established the legal framework for the medical treatment of mental disorder for detained patients. One of the protections for patients required by the Act is that once 3 months have elapsed since the person first received medication for mental disorder as a detained patient, medication may only be given if the treatment is authorised by either the clinician in charge of the patient's care (if the patient consents) or an independent doctor called a second opinion appointed doctor (SOAD) if the patient lacks capacity or refuses. The date from which this 3-month rule applies is called the consent to treatment (CTT) date. At the time of this study, for patients subject to a community treatment order (CTO) all medication had to be authorised by a SOAD and the time limit was 28 days. For electroconvulsive therapy (ECT) the authorisations were required from the start of the detention.

A SOAD is an independent psychiatrist sent at the request of the clinician in charge by the national body responsible for the SOAD service (the Care Quality Commission, CQC). This doctor will interview the patient, a nurse and another professional before deciding what medication (or ECT) may be given to the patient. The medication that may lawfully be prescribed for the patient must be described on the appropriate form, either T2 or T3 for detained in-patients or CTO1l for patients given a CTO. If 'urgent treatment' is required it may be authorised by any registered medical practitioner (Sections 62, 64B and 64G) subject to specific additional criteria. The Mental Health Act
Code of Practice states that 'urgent treatment' applies only if the treatment in question is immediately necessary to:

- save the patient's life;

- prevent a serious deterioration of the patient's condition, and the treatment does not have unfavourable physical or psychological consequences which cannot be reversed;

- alleviate serious suffering by the patient and the treatment does not have unfavourable physical or psychological consequences which cannot be reversed and does not entail significant physical hazard; or

- prevent patients behaving violently or being a danger to themselves or others, and the treatment represents the minimum interference necessary for that purpose, does not have unfavourable physical or psychological consequences which cannot be reversed and does not entail significant physical hazard. ${ }^{1}$

Although it is necessary to have a mechanism to enable patients to receive treatment that is 'immediately necessary', treatment given under these provisions is not subject to the same independent safeguard as that prescribed with the authority of a SOAD. Although previous similar studies by Johnson \& Curtice and Haw \& Shanmugarutnum highlighted that the most common reasons for the use of urgent treatment provisions were ECT and rapid tranquillisation, ${ }^{2,3}$ there was a general feeling among the practising clinicians that these provisions were being more commonly used for the complete treatment plan waiting for authorisation by the SOAD after the CTT date applied, in an 
attempt to provide lawful treatment. We attempted to find out whether this is actually the case.

The aim of our study was to understand the circumstances in which Sections 62 and 64B/G are used in clinical practice and to confirm, or otherwise, compliance with the Mental Health Act 1983. Also, we aimed to see whether the urgent treatment was required as a result of delay in assessment by a SOAD, and if so, to consider the cause of the delay.

\section{Method}

The study was conducted retrospectively covering a 1-year period from January 2009 to December 2009. A list of patients detained under Sections 62 and $64 \mathrm{~B}$ during the study period was obtained electronically from the information technology department. Complete lists of patients for whom forms $\mathrm{T} 2$ or $\mathrm{T} 3$ were filled in and patients commencing treatment under a CTO were also obtained electronically. Section 64 and CTO1l forms were obtained from the Mental Health Act office of the trust. Respective case notes were then located and more detailed information was obtained on the circumstances of use of Sections 62 and 64B/G.

\section{Results}

There were 58 instances of use of Section 62, in 39 patients, in the study period of 1 year (this was because Section 62 was used on more than one occasion for some patients). For Section $64 \mathrm{~B} / \mathrm{G}$, a total of 32 instances were identified in 26 patients. Demographic details of the patient population in each group are detailed in Table 1.

\section{Reasons documented}

The reasons documented on the urgent treatment form, detailing the need to use the respective provisions, are summarised opposite.

\begin{tabular}{|c|c|c|}
\hline & Section 62 & $\begin{array}{l}\text { Section } \\
64 B / G\end{array}$ \\
\hline \multicolumn{3}{|l|}{ Use of section, $n$} \\
\hline Total use & 58 & 32 \\
\hline Patients & 39 & 26 \\
\hline Case notes located & 38 & 22 \\
\hline \multicolumn{3}{|c|}{ Demographic characteristics } \\
\hline Age, years: mean & 46.5 & 43.5 \\
\hline \multicolumn{3}{|l|}{ Gender, $n(\%)$} \\
\hline Male & $25(64)$ & $20(77)$ \\
\hline Female & $14(36)$ & $6(23)$ \\
\hline \multicolumn{3}{|l|}{ Ethnicity, $n(\%)$} \\
\hline White British & $26(89)$ & $16(50)$ \\
\hline Asian & $9(20)$ & $8(25)$ \\
\hline Black Caribbean & $2(5)$ & $6(19)$ \\
\hline Mixed & $2(5)$ & $1(3)$ \\
\hline Other & $5(11)$ & $1(3)$ \\
\hline
\end{tabular}

Section 62

On 26 occasions (45\%) Section 62 was used because of a delayed SOAD assessment. On 18 occasions (31\%) it was used to prevent serious deterioration of the patient's condition, 9 times (15\%) to prevent the patient acting violently, twice (3\%) to save the patient's life and once (2\%) to alleviate serious suffering by the patient. On one form the reason for use was not documented. Details were not obtained from another form as the case notes were not located.

\section{Section $64 B / G$}

Section $64 \mathrm{~B} / \mathrm{G}$ was used on 25 occasions $(78 \%)$ to prevent serious deterioration of the patient's condition, 6 times (19\%) because of delayed SOAD assessment and once (3\%) to alleviate serious suffering by the patient.

\section{Need for urgent treatment}

The need for urgent treatment refers to the detailed circumstances as to why the urgent treatment provision was required, as concluded from detailed scrutiny of the case notes. Figures 1 and 2 detail different circumstances of use of emergency treatment provisions of the Mental Health Act.

\section{Section 62}

In 26 instances (45\%) Section 62 was used for the full treatment plan while waiting for the SOAD. On 11 occasions (19\%) it was used to change medications on an already approved treatment plan. Three instances (5\%) occurred when Section 62 had to be used to add medications which were erroneously missed on a T3 form by the SOAD. On one occasion (2\%) it was used to exceed the authorised maximum dose of a certified medication. In six instances $(10 \%)$ it was used to administer ECT. On another six occasions (10\%) it was required for new, urgent medication (rapid tranquillisation). On four occasions (7\%) it had to be used when patients treated in accordance with a T2 form withdrew their consent.

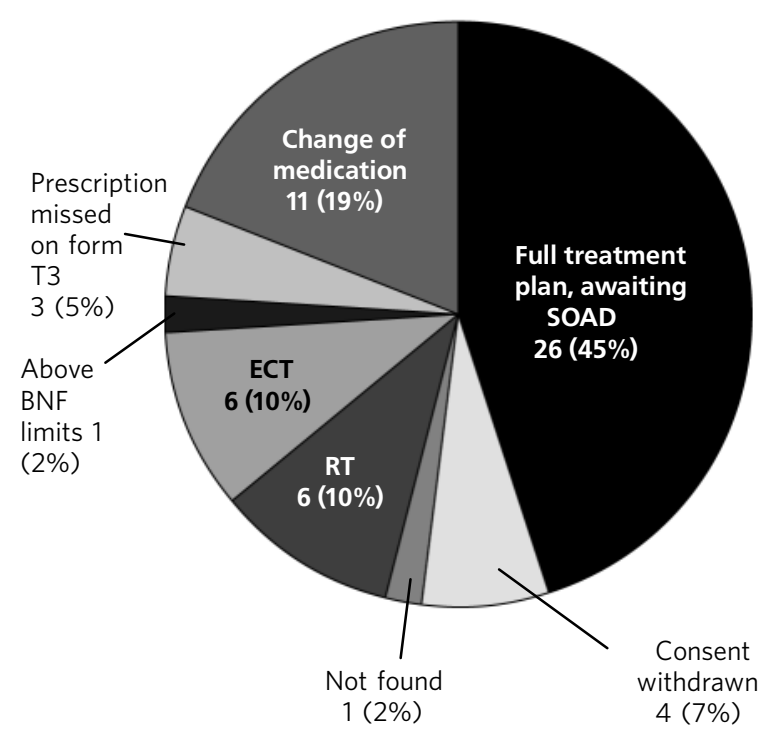

Fig 1 Need for urgent treatment under Section 62. BNF, British National Formulary; ECT, electroconvulsive therapy; RT, rapid tranquillisation; SOAD, second opinion appointed doctor. 


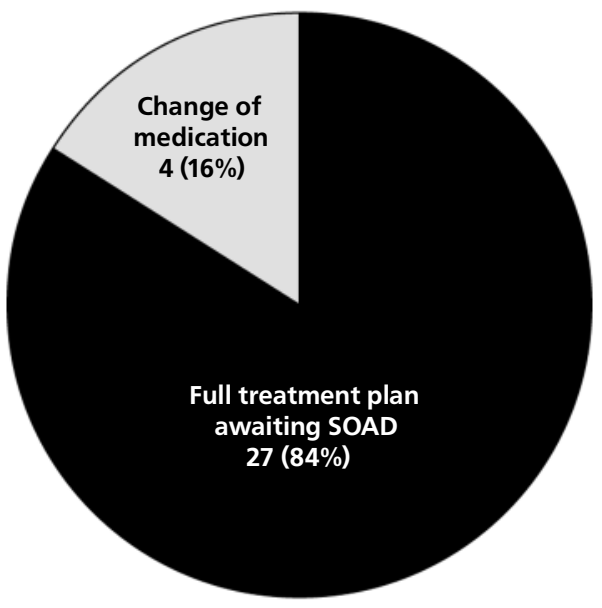

Fig 2 Need for urgent treatment under Sections 64 B and G. SOAD, second opinion appointed doctor.

\section{Section 64B/G}

The most common reason for use of Section $64 \mathrm{~B} / \mathrm{G}$ was for a full treatment plan while waiting for the $\operatorname{SOAD}(n=27$, $84 \%)$. The other five times (16\%) it was used to change medications on an already authorised treatment plan.

\section{Interventions}

Section 62 was used on $51(88 \%)$ occasions to administer medication and on $6(10 \%)$ occasions to administer ECT. The medications used were oral antipsychotics (28\%), anxiolytics (21\%), antimanics (14\%), depot antipsychotics (11\%), hypnotics (9\%), antidepressants (5\%), anticholinergics (2\%), zuclopenthixol acetate $(5 \%)$ and others (5\%). Section $64 \mathrm{~B}$ was used in all instances $(n=32,100 \%)$ to administer medication. The medications used were oral antipsychotics (27\%), depot antipsychotics (18\%), antimanics (14\%), anticholinergics (16\%), anxiolytics (12\%), hypnotics (7\%), antidepressants (4\%), zuclopenthixol acetate (5\%) and others $(2 \%)$.

\section{Full treatment plan awaiting SOADs}

There were 26 instances of the use of Section 62 and 27 instances of the use of Section $64 \mathrm{~B} / \mathrm{G}$ for a full treatment plan while waiting for a SOAD. Waiting times for these are detailed in Table 2.

\section{Use of urgent treatment provisions for ECT}

Urgent treatment provisions were used on six occasions for administering ECT. The date of request to SOADs was found in four case notes: the mean elapsed time between sending the SOAD request and receiving authorisation for ECT was 10.5 days (s.d. $=5.80,95 \%$ CI $1.27-19.73$; median 10.5, range 5-16).

\section{Discussion}

Our study indicates that urgent treatment provisions are increasingly being used for a full treatment plan (medication) while waiting for a SOAD to authorise the plan. This is in contrast to previous studies where ECT or rapid tranquillisation were the most common interventions used under Section 62. ${ }^{2,3}$ Although Haw \& Shanmugarutnum did not indicate that any of these instances were due to delay in the SOAD authorising the treatment, ${ }^{3}$ Johnson \& Curtice indicated that in $18 \%$ of their cases the urgent treatment provision was used to administer ECT owing to a delay in obtaining SOAD authorisation. ${ }^{2}$ However, neither study indicated that the urgent treatment provision was used for a full treatment plan while awaiting SOAD approval.

Although it may be argued that one of the reasons for delay in SOAD treatment authorisation could be a delay in sending the request for the same, it does appear from our study that the requests for SOADs were sent on average 5.33 days before the CTT date. Also, although the CQC states that it aims to arrange a visit within 5 working days from receipt of the request made for medications and within 2 working days for $\mathrm{ECT}^{4}$, our study shows that this period on average is 22 days for medications and 10.5 days for ECT. The CTO11 forms were received on average 21.4 days after the CTT date.

It is recognised that there is an increased demand for statutory second opinions, particularly since the introduction of CTOs in November 2008 and the requirement that all patients with a CTO should have a SOAD-authorised treatment plan. ${ }^{1}$ This applies regardless of whether the patient concerned is capacitous and consenting. Statistics from England and Wales show that 4107 CTOs were made during 2009-2010. ${ }^{5}$ Furthermore, between their introduction in November 2008 and the end of March 2010, a total of 6241 CTOs were made - an average of 367 a month. This is in addition to 45755 hospital detentions during the same period. ${ }^{5}$ In $2009-2010$ the CQC received

\begin{tabular}{|c|c|c|c|c|}
\hline & \multicolumn{4}{|c|}{ Time, days } \\
\hline & Mean & Median & Minimum & Maximum \\
\hline \multicolumn{5}{|l|}{ Section $62(n=26)$} \\
\hline Time between SOAD request and authorisation & 22 & 21 & 4 & 49 \\
\hline Time between CTT date and SOAD request & $-5.33^{\mathrm{a}}$ & 0 & $-22^{a}$ & 2 \\
\hline \multicolumn{5}{|l|}{ Section 64B/G $(n=27)$} \\
\hline Time between CTT date and CTO11 form ${ }^{\mathrm{b}}$ & 21.38 & 20 & $-10^{a}$ & 69 \\
\hline
\end{tabular}

CTO, community treatment order; CTT, consent to treatment; SOAD, second opinion appointed doctor.

a. Negative numbers indicate number of days before the CTT date.

b. Form CTO11 contains details of the authorised treatment plan for a patient on CTO. There were insufficient data with respect to the dates when the request for the SOADs for the CTOs were made. 
8781 requests for a SOAD to certify medications. ${ }^{5}$ This is $6 \%$ fewer requests overall compared with 2008-2009. However, services' use of urgent treatment powers to authorise medication has risen significantly. In 2004-2005 only $6 \%$ of patients had been given medication under urgent treatment powers before the SOAD visit; in 2009-2010 this figure had increased to $21 \%$ of patients referred for a second opinion. ${ }^{5}$ Whether this is due to the increased number of SOAD requests or other difficulties with the CQC - or both - we cannot say.

It is arguable whether administering a full treatment plan while waiting for SOAD authorisation fulfils either the spirit or the letter of the urgent treatment criteria of the Mental Health Act. The evidence suggests that the Act's safeguards, in relation to medication, and perhaps ECT, are being bypassed by use of the 'urgent treatment' provisions because of the non-availability of SOADs. Despite the CQC's reported decline in requests for SOADs in 2009-2010, ${ }^{5}$ practising clinicians and SOADs have undoubtedly felt that the $\mathrm{CQC}$ workload with respect to SOAD examination has increased markedly since CTOs were introduced. This raises two separate, but interrelated, issues. First, given the decrease in the total number of SOAD requests, has SOAD availability decreased? If so, why and what can be done about it? This is outside the scope of this paper. Second, can the requirement for SOADs be reduced without significant damage to the safeguards? At the time of this study all patients prescribed medication for mental disorder under a CTO required a SOAD certificate, whereas detained patients only required a SOAD certificate if they refused, or lacked capacity to consent to, the treatment. The CQC stated that consenting patients on a CTO accounted for about $45 \%$ of the requests they received for SOAD relating to CTOs. ${ }^{5}$ It seems odd to have greater protection for patients on a CTO than for those detained in hospital. This anomaly has now been corrected so that capacitous consenting patients on a CTO do not now require a SOAD certificate.

\section{Study implications}

Our study suggests that the urgent treatment provisions of the Mental Health Act 1983 are increasingly being used for a full treatment plan while awaiting SOAD examination. This is perhaps unlawful use of the urgent treatment provisions and is certainly outside the guidance of the Code of Practice. ${ }^{1}$ In addition, the availability of SOADs may need to be increased or the circumstances for the legal requirements for a $\mathrm{SOAD}$ be reduced. The latter may now have been achieved by permitting responsible clinicians to authorise consent to treatment for capacitous consenting patients subject to a CTO as they do for detained in-patients.

\section{About the authors}

Dr Rashmi Yadav is an ST5 trainee in general adult psychiatry in Leeds Partnership NHS Foundation Trust. Dr Anthony S. Zigmond is a consultant psychiatrist in Leeds Partnership NHS Foundation Trust and an honorary senior clinical lecturer at the University of Leeds.

\section{References}

1 Department of Health. Code of Practice: Mental Health Act 1983. TSO (The Stationery Office), 2008.

2 Johnson I, Curtice M. Use of Section 62 in clinical practice. Psychiatr Bull 2000; 24: 154

3 Haw C, Shanmugarutnum R. Use of Section 62 in clinical practice. Psychiatr Bull 2000; 24: 276.

4 Care Quality Commission. Guidance Note for Commissioners on Consent to Treatment and the Mental Health Act 1983, October 2008 (Amended September 2009). CQC, 2009.

5 Care Quality Commission. Mental Health Act Annual Report 2009/10. CQC, 2010. 